\title{
The Prevalence and Incidence of Clinical and Asymptomatic Lyme Borreliosis in a Population at Risk
}

Heinz Fahrer, Sjef M. van der Linden, Marie-Josephe Sauvain, Lise Gern, Elyes Zhioua, and André Aeschlimann

\author{
Department of Rheumatology, University of Berne, and Department of \\ Zoology, University of Neuchâtel, Switzerland; Department of \\ Medicine, Division of Rheumatology, University of Limburg, \\ Maastricht, Netherlands
}

\begin{abstract}
A past history of clinical Lyme borreliosis and the 6-month incidence of clinical and asymptomatic Lyme borreliosis was studied prospectively in a high-risk population. In the spring, blood samples were drawn from 950 Swiss orienteers, who also answered a questionnaire. IgG antiBorrelia burgdorferi antibodies were detected by ELISA. Positive IgG antibodies were seen in $248(26.1 \%)$, in contrast to $3.9 \%-6.0 \%$ in two groups of controls $(n=101)$. Of the orienteers, $1.9 \%-3.1 \%$ had a past history of definite or probable clinical Lyme borreliosis. Six months later a second blood sample was obtained from 755 participants, $558(73.9 \%)$ of whom were seronegative initially; $45(8.1 \%)$ had seroconverted from negative to positive. Only $1(2.2 \%)$ developed clinical Lyme borreliosis. Among all participants, the 6-month incidence of clinical Lyme borreliosis was $0.8 \%$ (6/755) but was much higher $(8.1 \%)$ for asymptomatic seroconversion (45/558). In conclusion, positive Lyme serology was common in Swiss orienteers, but clinical disease occurred infrequently.
\end{abstract}

Lyme disease, now often called Lyme borreliosis (LB), may affect the skin, heart, joints, and nervous system [1-6]. Neurologic, cardiologic, and rheumatologic manifestations such as meningoencephalitis, neuropathy, atrioventricular nodal block, myocarditis, and arthritis may follow in a few weeks to months after erythema migrans (EM). This skin lesion, although characteristic, is by no means an obligatory sign of LB; it was found in only $41 \%-61 \%$ of patients with the disease $[7,8]$. Arthritis typically is oligoarticular and recurrent but may become chronic and erosive; it predominantly involves the knee joints $[3,9]$.

LB is caused by the bite of ticks infected with the spirochete Borrelia burgdorferi $[10,11]$. The ticks that can transmit disease are part of the Lxodes ricinus complex. The discovery of the spirochete led to serology as an aid in establishing the aiagnosis of LB, especially in the absence of EM [12].

In Europe the disorder seems to have a wide and constant distribution [13]. In contrast, in the United States LB may have been confined to three enzootic areas (northeast coast, midwest, California), but cases appear to be increasing and infaction to be spreading to new areas. Recognition may also accisunt for some of the apparent increase. LB has now been reported from 35 states.

\footnotetext{
Received 13 January 1989, revised 20 June 1990.

This work constitutes a part of the thesis of E.Z.

Informed consent was obtained from participants.

Grant support: Swiss National Research Fund (3.892-0.86).

Reprints or correspondence: Dr. Sjef van der Linden, University Hospital, P.O. Box 1918, NL-6201 BX Maastricht, Netherlands.

The Journal of Infectious Diseases 1991;163:305-310

(C) 1991 by The University of Chicago. All rights reserved.

0022-1899/91/6302-0015\$01.00
}

In Long Island, New York, the incidence of LB was found to be considerably higher than previously recognized [14]. Some patients showed inapparent seroconversion after infection. The estimated ratio of apparent to inapparent infection was 1:1 in a study in Great Island, Massachusetts [8].

To assess the usefulness of Lyme serology as a diagnostic tool, it would be important to know not only the sensitivity and specificity of the test but also its predictive value. This would imply knowledge about the prevalence and incidence of clinical LB and the ratio of apparent to inapparent infections in populations where the test is applied.

We studied past history of clinical LB and frequency of positive Lyme serology in a population at risk and the frequency of LB manifestations and seroconversion during 6 months of follow-up.

\section{Methods}

Orienteers from all parts of Switzerland were invited to participate in the study. Orienteers, using map and compass, run competitively through rough brushy terrain, the preferred habitat of $I$. ricinus. Often running in short pants and sport shirts, they are at risk of being bitten by ticks. Orienteers usually exercise regularly in the forests close to their homes. In addition, they participate every year in national competitions held in spring and autumn in various brushy regions of Switzerland. In this country the infection rate of $I$. ricinus with $B$. burgdorferi ranges from $5 \%$ to $34 \%$ [15].

Study group. Blood $(10 \mathrm{ml})$ was obtained by venipuncture from the participants of the 1986 spring competitions. Participants were also asked to complete, on site, a questionnaire about exposure to ticks and possible manifestations of LB. The following autumn, a second blood sample was taken, and again a questionnaire was given to the participants. Posters showing photographs of a typical example of EM and of a tick attached to normal skin were shown to all 
orienteers on both occasions. The participants were encouraged to discuss any problems associated with answering the questionnaire with the study team. They were further asked to report health problems arising during the follow-up period immediately to one of us (H.F.). An English summary of the questions, originally posed in French and German, is available on request.

If the answers on the questionnaire suggested possible LB (e.g., redness of the skin or neurologic or joint symptoms), further information was obtained by a telephone interview. Disease features were defined as probable evidence for LB if the history strongly suggested LB but a physician had not been consulted. Only disease manifestations confirmed by a physician were considered definite evidence for LB. Review of the clinical data was done by two of us (H.F., M.S.) without access to the serologic results.

A total of 70 patients had seen a physician because of signs or symptoms compatible with a diagnosis of LB. A letter was sent to these physicians requesting the precise medical findings and details regarding diagnosis and effects of treatment. If, after this procedure, uncertainty as to the clinical diagnosis still persisted, a telephone call to the physician was made. Definite diagnoses of LB were based on physicians' judgments, but it was accepted that the diagnosis was established retrospectively. In case of arthritis, other causes (e.g., rheumatoid arthritis, ankylosing spondylitis, or reactive arthritis) had to be very unlikely, at least in retrospect. Neurologic manifestations were accepted as due to LB only if other explanations were considered unlikely.

Controls. To assess the specificity of a serologic test for LB, a reference standard for the absence of the disease and for exposure to $B$. burgdorferi is necessary. The diagnosis of LB can be based on clinical judgment or a specific set of diagnostic criteria, which allows also defining the absence of disease. However, in countries such as Switzerland it is impossible to definitely exclude exposure to $B$. burgdorferi, even if people deny LB manifestations and do not recall tick bites. Therefore, two different control groups were included in the study.

Sera were obtained from 51 healthy volunteers (blood donors) who had spent most of their life at altitudes $>1000 \mathrm{~m}$. . ricinus, the tick that transmits LB in Europe, is rare at $1000-1500 \mathrm{~m}$ and has not been found at altitudes $>1500 \mathrm{~m}$ [16]. Of course, these persons sometimes may have visited regions at lower altitudes, but on average they probably had a lower level of exposure to $B$. burgdorferi than did most Swiss people.

Demographic data (age, sex, profession) were not available for these 51. Therefore, a second control group was used: 50 healthy individuals, 18 male and 32 female, who lived in the greater Berne area, which has $>200,000$ inhabitants. The city is situated at an altitude of $500 \mathrm{~m}$. All gave a negative history for LB and denied tick bites. None had engaged in orienteering. The mean age $( \pm S D)$ in this group was $38.1 \pm 15.8$ years. One person was employed at a farm; the others were housewives or worked at an office or in a hospital.

Serology. After centrifugation sera were stored at $-20^{\circ} \mathrm{C}$ until testing for antibodies against $B$. burgdorferi. $B$. burgdorferi strain B31 was used as the source of antigen. An ELISA was done to detect IgG antibody to $B$. burgdorferi. Briefly, the ELISA antigen was prepared as described by Russell et al. [17]. Included in each run was one pooled negative control serum and a strongly positive test serum, which was diluted six times from 1:300 to 1:9600. The curve obtained was used as reference for study sera. The optical density (OD) of each sample was marked on this line and the result was expressed as the logarithm of the diluted positive test serum. (Note that low log-dilution values correspond with lesser dilution of the positive test serum and therefore represent high anti-Borrelia antibody levels, whereas high log-dilution values reflect low antibody levels.)

Each sample was tested in duplicate, and the mean value was calculated. If both values for one sample differed by $>10 \%$, the test was repeated. The second sample for each individual was tested $\sim 6$ months after the first sample.

In the absence of a reference standard, the positive cutoff point for IgG antibodies was defined as a log dilution of $\leqslant 3.74$ corresponding to 2 SD below the mean $\log$ dilution of the 51 sera of the people living at high altitude. This implies that statistically $\sim 2.5 \%$ of supposedly unexposed people might be expected to show false-positive test results (type I error). Taking this cutoff point, the sensitivity of the serologic test varied from $26 \%$ to $100 \%$ depending on the clinical manifestations in cases of definite LB: EM, 26\% (7/27); neurologic, 74\% (31/42); arthritis, 93\% (13/14); and acrodermatitis chronica atrophicans, $100 \%(10 / 10)$.

Fifty-one other sera were also tested at the laboratory of the Department of Microbiology of the University of Limburg at Maastricht, Netherlands. There was good agreement between laboratories $(r=$ $.68 ; P=.0001$ ).

Statistics. $\chi^{2}$ analysis was applied to categorical data. In addition, a stepwise regression analysis was used to explain the variation in ELISA results. Included in the regression model as predictors were age and sex of the orienteers, the number of years practicing orienteering, the number of hours spent weekly in forests for training activities, and the number of recalled tick bites.

\section{Results}

Responders. Of a total of 1294 participants in several spring competitions, 964 agreed to enter the study (response rate, $74.4 \%$ ). Of them, $617(64 \%)$ were male and 347 female. The mean age $( \pm S D)$ of the responders was $31 \pm 14$ years (range, 10-74). In the fall, a second serum sample was obtained from 755 (78.3\%) of the 964 participants.

Exposure rate. Most (78\%) of the 964 persons who entered the study reported at least one tick bite (table 1). There

Table 1. History of tick exposure related to prevalence of IgG anti-Borrelia antibodies (ELISA).

\begin{tabular}{lrrr}
\hline No. of bites & \multicolumn{2}{c}{$\begin{array}{c}\text { No. of } \\
\text { participants (\%) }\end{array}$} & $\begin{array}{r}\text { No. with positive } \\
\text { IgG serology (\%) }\end{array}$ \\
\hline Do not know & 88 & $(9.3)$ & $19(21.6)$ \\
Never bitten & 122 & $(12.8)$ & $22(18.0)$ \\
$1-5$ & 356 & $(37.5)$ & $80(22.5)$ \\
$6-10$ & 160 & $(16.8)$ & $53(33.1)$ \\
$11-50$ & 192 & $(20.2)$ & $04(33.3)$ \\
$>50$ & 32 & $(3.4)$ & $10(31.3)$ \\
$\quad$ Total & $950(100.0)$ & $248(26.1)$ \\
\hline
\end{tabular}


was only modest association between the serologic results and the number of tick bites recalled.

Prevalence of positive ELISA Lyme serology. IgG ELISA tests could be done for 950 study participants $(98.5 \%)$. Of the orienteers, $248(26.1 \%)$ reacted positively. Of controls, $3.9 \%(2 / 51)$ from high altitudes and $6.0 \%(3 / 50)$ from the greater Berne area were positive.

Prevalence of a history of Lyme manifestations related to Lyme serology. Complete clinical and serologic data were available for 950 (98.5\%); 18 (1.9\%) had had definite LB manifestations (table 2). In another 11, LB could not be confirmed by a physician, but patients' descriptions of their findings strongly suggested LB. These 11 cases were considered probable LB. Seven (39\%) of the 18 with definite LB had had no EM, 5 (28\%) had suffered from arthritis, and 3 $(17 \%)$ from neurologic manifestations. Therefore, in this highrisk group the prevalence of a history of clinical LB was $1.9 \%$ for definite disease (18/950) and 3.1\% for definite or probable disease (29/950). The probable LB cases included 6 who reported classic EM but did not consult a physician for it.

Clinical manifestations providing definite or probable evidence for LB were related to the results of ELISA antibody tests (table 2). Among the 18 with a definite history of LB, ELISA IgG anti-Borrelia antibodies were present in $9(50 \%)$. Of course, we do not know whether in the past a conversion from seropositive to seronegative occurred in the other 9 . Only 1 patient had been given antibiotic treatment for LB. However, we do not know how many patients may have received such treatment for unrelated health problems.

Incidence study. Altogether, $69(12.4 \%)$ of 558 participants negative for IgG by ELISA in the spring were positive in the autumn (table 3); 29 of these noticed at least one tick bite between spring and autumn. The 69 who showed seroconversion included some borderline cases (figure 1). Therefore, how much change on the log-dilution scale in the IgG ELISA technique represents relevant change? To put it differently, how much change correlates with a fourfold rise in immunofluorescence titer, which is taken as the usual standard of clinical significance? In our hands, a decrease in $\log$ dilution of 0.40 roughly corresponds to a fourfold increase in immunofluorescence titer (unpublished data).

A total of 63 subjects seronegative in the spring had a decrease in log dilution of at least 0.40 . In 45 of them the autumn sample showed true seroconversion, as the follow-up $\log$-dilution value was at or below the 3.74 cutoff point. Therefore, $45(8.1 \%)$ of the initially seronegative participants both showed significant decrease in log-dilution values and passed the cutoff value of 3.74 . In 17 of the other 18, the follow-up value was between 3.75 and 4.00 , that is, close to positivity. Only one of these 63 subjects who showed significant increase in specific IgG antibody developed LB manifestations during the 6 months between sampling: This person developed Bell's palsy and had an IgG ELISA decrease from 3.99 to 2.62.
Table 2. Definite and probable Lyme borreliosis features related to results of ELISA anti-Borrelia antibody tests.

\begin{tabular}{ll}
\hline Type of borreliosis, symptom(s), year & ELISA IgG \\
\hline Definite & \\
Polyarthritis, 1970-1972 & 2.07 \\
Arthritis knee, 1983 & 2.84 \\
EM, 1980 & 2.90 \\
EM, 1976 & 3.01 \\
EM, 1985 & 3.14 \\
EM, 1985 & 3.16 \\
Meningitis, 1960 & 3.54 \\
Arthritis, 1978 & 3.64 \\
EM, meningitis, 1983 & 3.71 \\
Meningitis, 1978 & 3.76 \\
Arthritis, 1976 & 3.83 \\
EM, 1985 & 4.01 \\
EM, 1984 & 4.05 \\
Polyarthritis, 1985 & 4.10 \\
EM, 1979 & 4.13 \\
EM, 1976 (penicillin treatment) & 4.16 \\
EM, 1986 & 4.23 \\
EM, 1977 & 4.35 \\
Probable & \\
EM, 1984 & 2.66 \\
Meningitis, 1976 & 2.99 \\
Meningitis, Bell's palsy, 1974 & 3.15 \\
EM, 1975 & 3.24 \\
EM, 1985 & 3.77 \\
EM, 1985 & 3.97 \\
Arthritis, 1981 & 4.03 \\
EM, 1983 & 4.23 \\
Lymphadenosis cutis benigna, 1982 & 4.27 \\
Arthritis, 1973 & 4.29 \\
& 4.33 \\
\hline
\end{tabular}

NOTE. Positive cutoff: $\log$ dilution of $\$ 3.74$. EM $=$ erythema migrans.

Table 3. ELISA IgG anti-Borrelia burgdorferi antibodies in autumn compared with presence of antibodies in the spring of the same year.

\begin{tabular}{lccc}
\hline & \multicolumn{2}{c}{ Spring IgG antibody } & \\
\cline { 2 - 3 } Autumn & Positive & Negative & Total \\
\hline IgG antibody & 153 & 69 & 222 \\
Positive & 44 & 489 & 533 \\
Negative & 197 & 558 & 755 \\
\hline
\end{tabular}

Clinical features of LB were also observed among 5 orienteers who did not show seroconversion. Three persons had positive IgG tests before they developed EM. On the other hand, definite LB features during follow-up were seen in 2 who had negative IgG antibody tests both in the spring and fall. One of these developed classic EM in the summer. The other, who had borderline ELISA results on both occasions, showed an atrophic skin lesion of the trunk due to $B$. burgdorferi. This lesion is known by dermatologists as atropho- 


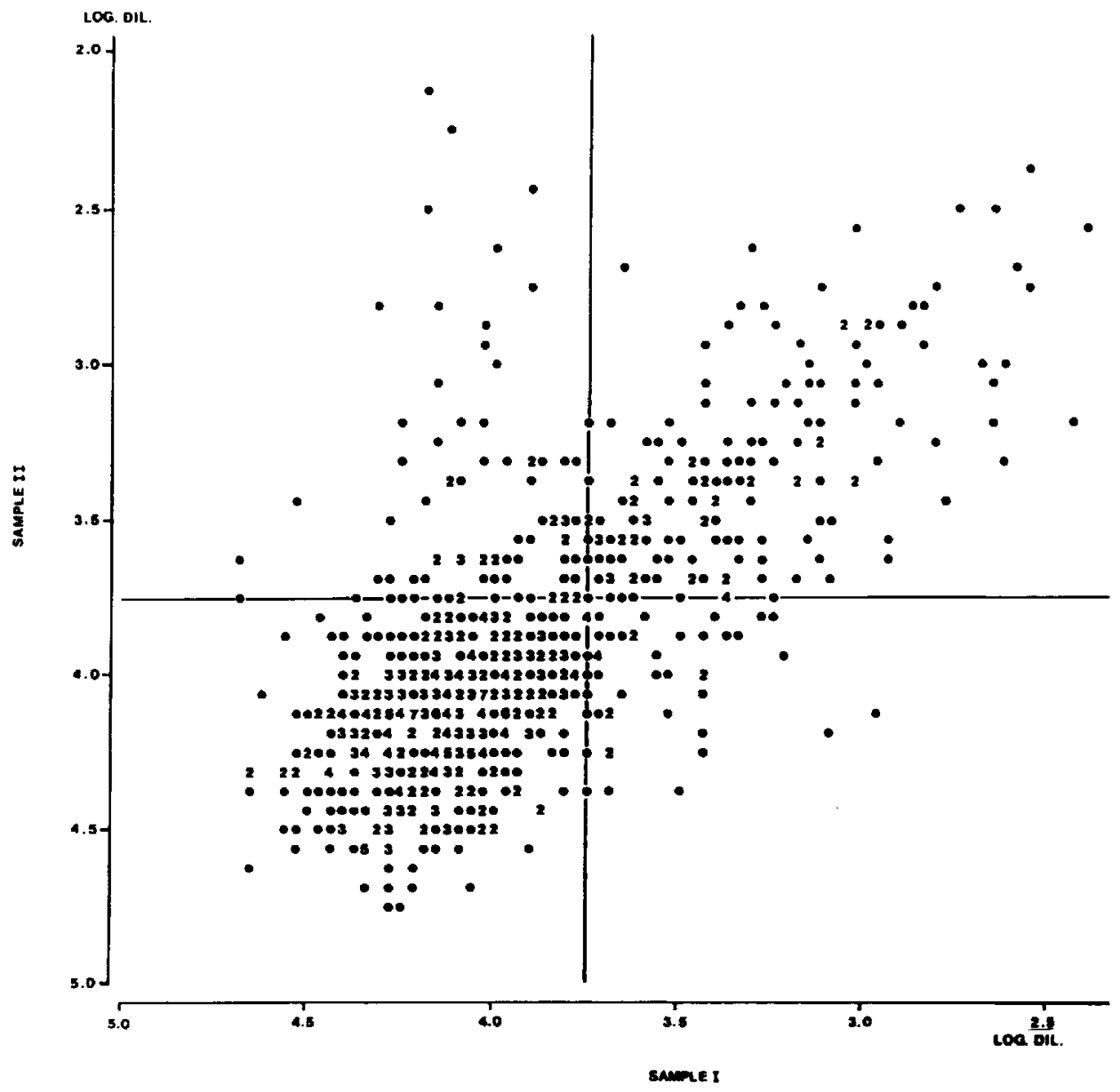

Figure 1. Plot of IgG ELISA tests for anti-Borrelia burgdorferi antibodies in serum samples obtained in the spring (sample I) and autumn (sample II). Cutoff for a positive IgG ELISA is $\log$ dilution $\leqslant 3.74$. derma of Pasini and Pierini. All patients were treated with penicillin.

Thus, in total 6 of 755 orienteers developed definite LB features in the 6 months between spring and fall. Hence, the 6-month incidence was $0.8 \%$ for clinical LB but much higher $(45 / 558,8.1 \%)$ for inapparent infection.

Regression analysis. Only the number of tick bites and age contributed significantly to explaining the variation in ELISA serology; however, the amount of variance was small $\left(\mathrm{R}^{2}=.03\right)$. Gender, the number of years practicing orienteering, and the number of hours spent every week in forests did not contribute significantly.

\section{Discussion}

Determining a valid estimate of the prevalence and incidence of a disease requires reliable diagnostic tools. The problem in defining specificity in an infectious disease such as LB is that currently no reference standard for the absence of exposure is available. Therefore, in individual cases it is impossible to differentiate asymptomatic (subclinical) infection from false-positive serology if syphilis or other spirochetoses have been ruled out. To minimize this problem we used as one control group blood donors who, because they lived at high altitudes, supposedly had had low exposure to $B$. burgdorferi.

The most striking feature from this study is the discrepancy between the high prevalence and incidence of positive Lyme serology and the infrequent development of clinically apparent LB. However, in the interpretation of these findings the possibility of four sources of bias should be considered.

First, our serologic tools could have been too sensitive, resulting in a high proportion of false-positives. This explanation, however, seems unlikely, since at the chosen cutoff level the increased prevalence ( $26.1 \%$ ) of high antibody titers occurred exclusively in the orienteers, but not in the controls (prevalence, $3.9 \%-6.0 \%$ ). This means that orienteers were 4.4-6.7 times more likely to have positive serologic results. The low prevalence of positive antibodies in the high-altitude controls supports a favorable specificity of the test. Also, the $4 \%-6 \%$ prevalence of antibodies is of the same magnitude as found in other studies (discussed below). Although the 50 controls from the Berne area denied having had tick bites, this does not exclude exposure to $B$. burgdorferi, because infection may have occurred unnoticed. This is also shown in 
table 1 , in which $12.8 \%$ of those who denied ever being bitten had positive Lyme serology. This raises questions about the discriminative value of this part of the clinical history as a diagnostic tool for the diagnosis of LB and about the usefulness of recalled tick bites as a classification or diagnostic criterion.

Raising the positive cutoff level of 3 (instead of 2) SD above the mean in the controls would increase the specificity, but the sensitivity would decrease. This phenomenon might partly explain why Dattwyler et al. [18] found some patients with definite LB to have negative serologic tests but at the same time to show positive stimulation of lymphocytes if challenged with $B$. burgdorferi antigen.

Second, LB features may have been underreported by orienteers. Also, the validity and reliability of the questionnaire was not assessed beforehand. However, overreporting of such features of LB as skin lesions, joint problems, or possible neurologic complications seems more likely than underreporting. EM especially may be regarded as a reliable feature, because it is easily noticed and has characteristics that make it likely to be recalled by a patient. Typical examples of EM were shown on a poster to all participants.

Third, interpretation of reported features as compatible with LB might have been too restrictive. However, even if we were to accept every reported clinical feature as proof of $\mathrm{LB}$, most of those with anti- $B$. burgdorferi antibodies would have to be classified as having an asymptomatic disease: Only $28 \%$ with IgG antibodies reported clinical features in the questionnaire. Therefore, the absence of LB manifestations in most seropositives is indeed striking.

Fourth, we could have missed recent or unimpressive cases. The 6-month follow-up study indicated that only $1(1.6 \%)$ of 63 who showed a significant increase in IgG antibodies against B. burgdorferi developed signs of LB within the study period. However, some recently infected people could already have developed positive serology before the appearance of clinical manifestations (i.e., further follow-up is indicated). Also, it is possible we missed some LB cases who had nonspecific symptoms such as fever, myalgia, or mild arthralgia only [19]. Such features may have passed unnoticed or have been forgotten by the orienteers. Nonetheless, based on our findings, it seems likely that - at least in Switzerland - most people infected with $B$. burgdorferi do not develop clinical manifestations of skin, joint, or nervous system. Therefore, in this respect, LB seems to resemble other infectious diseases as rubella or toxoplasmosis in which many infected persons do not develop arthritis or neurological manifestations. It is unclear to what extent such complications of the disease are due to host factors and to geographic differences within strains of $B$. burgdorferi. It has been suggested that some strains might be more pathogenic than others, possibly because of the presence of certain not-yet-identified plasmids. It is possible that variation in extrachromosomal DNA accounts for effects that determine the difference in disease expression among $B$. burg- dorferi microorganisms [20]. In general, European isolates have been more diverse than American isolates [6].

How do our data compare with other reports? Among rural West Germans, $15.7 \%$ of subjects had an immunofluorescent antibody IgG titer $\geqslant 1: 128$ [21-23], whereas $27 \%$ of forest workers and $46 \%$ of children who had tick bites had IgG titers $\geqslant 1: 64$ [24]. In England, positive serology figures ranging from $1 \%$ (London) to $7 \%$ (Southampton) were reported [25]. In the United States, the proportion of controls with positive Lyme serology in several studies ranged from none to $10 \%$ $[10,14,17,26,27]$. In an East Coast community, $4(3.1 \%)$ of 129 who were followed during the summer season showed at least fourfold rises in IgG antibody titer, including 2 without clinical LB [14].

The estimated 1:1 ratio of apparent to inapparent infection in two United States studies contrasts sharply with our results, which indicate that in Switzerland most infections remain inapparent $[8,14,28]$ The reason for the discrepancy is unclear. Possibly geographic differences in the pathogenicity of Borrelia strains or in the distribution of pathogenic strains should be taken into consideration. Therefore, our results may not necessarily be extrapolated to other countries. Clearly, more studies are needed. Remarkably, the difference in the incidence of clinical LB is less striking. Six persons from the cohort of 755 participants $(0.8 \%)$ developed LB between spring and autumn. Assuming that about the same number of cases could occur within the next half-year leads to an annual incidence of $\sim 12 / 755(1.6 \%)$. (The assumption that the same number of cases would occur between autumn and spring may slightly overestimate the true incidence.) This estimated incidence compares well with estimates in the United States, which range from 0 to $3.3 \%$ per year for two East Coast regions well known for the occurrence of $\mathrm{LB}[8,14]$.

What are the implications of the high prevalence of positive Lyme serology and the low occurrence of clinical disease? Serologic tests for LB are useful in the absence of EM or in diagnosis of late manifestations, which cannot be distinguished clinically from a variety of other diseases. Certainly, cautious interpretation of cutoff levels and titers is warranted $[29,30]$. But, can positive tests be taken a step beyond proof of infection and be used to establish a cause-and-effect relationship between the infection and clinical manifestations [31]? Our study strengthens the view that, at least in populations with a low prevalence of clinical LB and a high prevalence of asymptomatic positive Lyme serology, diagnostic testing is of limited value. If, in the absence of other evidence for LB, the pretest probability of LB is low, serologic testing will not offer any great advantage. This would be the case in a patient with unspecified arthritis who gives a negative history of EM. Indeed, symptoms caused by another disease may be attributed wrongly to LB [6].

Serologic testing is most useful if the pretest probability is $\sim 40 \%-60 \%$, for example, a patient with unspecified arthritis, who does not recall having had EM but has a history 
of meningitis or neuritis of unknown etiology before onset of joint symptoms $[32,33]$. It cannot be emphasized too strongly that serologic results must be interpreted with caution; the physician must beware of false-negative and, more commonly, false-positive results [6]. The decision to treat should be made with reference to the patient's clinical presentation and the predictive value of the diagnostic assay [34]. The results of serologic testing should not be relied on as the sole criterion in making the diagnosis of LB [35].

In conclusion, positive serology for LB was common in Swiss orienteers but clinical LB occurred infrequently. In such a setting, serologic diagnostic tests for anti-B. burgdorferi antibodies are of limited value in the absence of sufficient clinical evidence of LB.

\section{Acknowledgment}

We thank M. A. Khan for critically reading the manuscript and providing helpful comments.

\section{References}

1. Steere, AC, Malawista SE, Snydman DR, et al. Lyme arthritis: an epidemic of oligoarticular arthritis in children and adults in three Connecticut communities. Arthritis Rheum 1977;20:7-11.

2. Steere AC, Malawista SE, Hardin JA, Askenase PW, Andiman WA. Erythema chronicum migrans and Lyme arthritis. The enlarging clinical spectrum. Ann Intern Med 1977;86:685-98.

3. Steere AC, Schoen RT, Taylor E. The clinical evolution of Lyme arthritis. Ann Intern Med 1987;107:725-31.

4. Reik L, Steere AC, Bartenhagen NH, Shope RE, Malawista SE. Neurologic abnormalities of Lyme disease. Medicine (Baltimore) 1979; 58:281-94.

5. Steere AC, Batsford WP, Weinberg M, et al. Lyme carditis: cardiac abnormalities of Lyme disease. Ann Intern Med 1980;93:8-16.

6. Steere AC. Medical progress: Lyme disease. N Engl J Med 1989;321: 586-96.

7. Schmidt R, Kabatzki J, Hartung S, Ackermann R. Erythema migrans Borreliosis in der Bundesrepublik Deutschland. Dtsch Med Wochenschr 1985;110:1803-7.

8. Steere AC, Taylor E, Wilson ML, Levine JF, Spielman A. Longitudinal assessment of the clinical and epidemiological features of Lyme disease in a defined population. J Infect Dis 1986;154:295-300.

9. Kryger $\mathrm{P}$, Hansen $\mathrm{K}$, Vinterberg H, Pedersen FK. Lyme borreliosis among Danish patients with arthritis. Scand J Rheumatol 1990;19:77-81.

10. Steere AC, Grodzicki RL, Kornblatt AN, et al. The spirochetal etiology of Lyme disease. N Engl J Med 1983;308:733-40.

11. Benach JL, Bosler EM, Hanrahan JP, et al. Spirochetes from the blood of two patients with Lyme disease. N Engl J Med 1983;308:740-2.

12. Craft JE, Grodzicki RL, Steere AC. Antibody response in Lyme disease: evaluation of diagnostic tests. J Infect Dis 1984;149:789-95.

13. Schmid GP. The global distribution of Lyme disease. Rev Infect Dis 1985;7:41-50.

14. Hanrahan JP, Benach JL, Coleman JL, et al. Incidence and cumulative frequency of endemic Lyme disease in a community. J Infect Dis 1984;150:489-96.
15. Aeschlimann A, Chamot E, Gigon F, Jeanneret JP, Kesseler D, Walther C. Borrelia burgdorferi in Switzerland. Zentralbl Mikrobiol 1986; A263:450-8.

16. Aeschlimann A. Ixodes ricinus, Linné, 1758. (Ixodoidea;Ixodidae). Essai préliminaire de synthèse sur la biologie de cette espèce en Suisse. Acta Trop Basel 1972;29:321-40.

17. Russell H, Sampson JS, Schmid GP, Wilkinson HW, Plikaytis B. Enzymelinked immunosorbent assay and immunofluorescence for Lyme disease. J Infect Dis 1984;149:465-70.

18. Dattwyler RJ, Volkman DJ, Luft BJ, Halperin JJ, Thomas J, Golightly MG. Seronegative Lyme disease: dissociation of specific T- and B-lymphocyte responses to Borrelia burgdorferi. N Engl J Med 1988;319: $1441-6$.

19. Steere AC, Bartenhagen NH, Craft JE, et al. The early clinical manifestations of Lyme disease. Ann Intern Med 1983;99:76-82.

20. Hyde FW, Johnson RC. Genetic relationship of Lyme disease spirochetes to Borrelia, Treponema, and Leptospira spp. J Clin Microbiol 1984;20:151-4.

21. Paul H, Gerth HJ, Ackermann R. Infectiousness for humans of Ixodes ricinus containing Borrelia burgdorferi. Zentralbl Mikrobiol 1986; A263:473-6.

22. Schmidt R, Ackermann R, Kabatzki J, Hartung H. Epidemiologic aspects of erythema chronicum migrans disease in the Federal Republic of Germany. Zentralbl Mikrobiol 1986;A263:435-41.

23. Wilske B, Muenchhoff $P$, Schierz G, Preac-Mursic V, Roggendorf M, Zoulek G. Zur Epidemiologie der Erythema migrans: Nachweis von Antikoerpern gegen Schildzecken-spirochaeten bei Waldarbeitern in Oberbayern. Muench Med Wochenschr 1985;127:171-3.

24. Muenchhoff P, Wilske B, Preac-Mursic V, Schierz G. Antibodies against Borrelia burgdorferi in Bavarian forest workers. Zentralbl Mikrobiol 1986;A263:412-9.

25. Cooper C, Muhlemann MF, Wright DJM, Hutchinson CA, Amstrong R, Maini RN. Arthritis as manifestation of Lyme disease in England (letter). Lancet 1987;1:1313-4.

26. Mertz LE, Wobig GH, Duffy J, Katzmann JA. Laboratory Medicine. Mayo Clin Proc 1985;60:402-6.

27. Wilkinson HW. Immunodiagnostic tests for Lyme disease. Yale J Biol Med 1984;57:567-72.

28. Gern L, Frossard E, Walter A, Aeschlimann A. Presence of antibodies against Borrelia burgdorferi in a population of the Swiss plateau. Lyme borreliosis II. Zentralbl Mikrobiol 1989;18(suppl):321-8.

29. Magnarelli LA, Anderson JF, Chappell WA. Geographic distribution of humans, raccoons, and white-footed mice with antibodies to Lyme disease spirochetes in Connecticut. Yale J Biol Med 1984;57:619-26.

30. Wilkinson HW, Russell H, Sampson JS. Caveats on using nonstandardized serologic tests for Lyme disease (letter). J Clin Microbiol 1985;21: 291-2.

31. Duffy J, Mertz LE. Serologic testing for Lyme disease (editorial). Ann Intern Med 1985;103:458.

32. Khan MA, Khan MK. Diagnostic value of HLA-B27 testing in ankylosing spondylitis and Reiter's syndrome. Ann Intern Med 1982;96:70-6.

33. Sackett DL, Haynes RB, Tugwell P. Clinical epidemiology: a basic science for clinical medicine. Boston: Little, Brown, 1985:59-138.

34. Barbour AG. The diagnosis of Lyme disease: rewards and perils. Ann Intern Med 1989;110:501-2.

35. Luger SW, Krauss E. Serologic tests for Lyme disease: interlaboratory variability. Arch Intern Med 1990;150:761-3. 